12

\title{
In situ модификация и анализ состава и кристаллической структуры кремниевой мишени с помощью ионно-пучковых методик
}

\author{
(C) Ю.В. Балакшин, ${ }^{1}$ А.А. Шемухин, ${ }^{1}$ A.B. Назаров, ${ }^{1}$ А.В. Кожемяко, ${ }^{2}$ В.С. Черныш ${ }^{2}$ \\ ${ }^{1}$ Научно исследовательский институт ядерной физики им. Д.В. Скобельцына, \\ 119991 Москва, Россия \\ ${ }^{2}$ Московский государственный университет им. М.В. Ломоносова, фризический фракультет, кафедра фризической \\ электроники, \\ 119991 Москва, Россия \\ e-mail: balakshiny@gmail.com
}

(Поступило в Редакцию 25 января 2018 г. В окончательной редакции 31 марта 2018 г.)

Метод резерфордовского обратного рассеяния (РОР) в сочетании с каналированием широко распространен в области анализа состава и структуры материалов. Представлены описание и технические параметры экспериментальной линии, предназначенной для проведения in situ ионной имплантации и спектрометрии РОР. Приведены параметры анализирующего пучка, необходимые для достижения погрешности в несколько процентов при исследовании профилей распределения примесей и дефектов. Разрешение методики было оценено при помощи спектра альфа частиц, полученных при распаде ${ }^{239} \mathrm{Pu}$ и из РОР спектра от монокристалла кремния. Имплантация ионов $\mathrm{Xe}^{+}$с энергией $100 \mathrm{keV}$ в монокристалл кремния и РОР анализ мишеней в режиме каналирования были проведены без нарушения вакуумных условий. Были изучены профиль распределения внедренных атомов и профиль распределения дефектов в облученных монокристаллах.

DOI: $10.21883 / J T F .2018 .12 .46796 .41-18$

\section{Введение}

Спектрометрия резерфордовского обратного рассеяния (РОР) позволяет получать количественную и качественную информацию о химическом составе образца, информацию о его кристаллической структуре. Этот метод с середины XX века используется для определения состава мишеней и распределения элементов по глубине путем анализа энергетических спектров обратно рассеянных частиц.

Метод использовался для изучения роста оксидных пленок на нержавеющей стали [1] и роста пленок $\mathrm{GaN}$ на кремнии при разработке экологически чистых источников энергии [2]. Так же при помощи метода РОР активно изучают дефектообразование при разработке материалов для оболочек ядерных реакторов [3], транзисторов с высокой электронной подвижностью [4], твердотельных лазеров [5]. В сочетании с каналированием метод используется при модификации оптических свойств кристалла для изучения влияния среза образца на дефектообразование [6]. Использование каналирования в обратном рассеянии продемонстрировано в работах, описывающих влияние параметров облучения на кристаллическую структуру и распределение дефектов в мишени типа „кремний-на-изоляторе“ [7-9]. Кроме того, метод широко применяется при изучении процессов диффузии. Например, в работе [10] при помощи РОР изучалась диффузия атомов золота в кремнии при разработке детекторов частиц высоких энергий. А в работе [11] было исследовано атомное перемешивание на границе раздела уран-молибденового сплава и алюми- ниевой матрицы для снижения потерь при обогащении ураном топливных элементов. При помощи метода РОР была обнаружена взаимная диффузия в зоне раздела между пленками $\mathrm{BiFeO}_{3}$ (BFO) и подложками ( $\mathrm{Si}\left(\begin{array}{lll}1 & 0 & 0\end{array}\right)$, $\mathrm{SiO}_{2}$ и $\mathrm{SiO}_{2} / \mathrm{Si}(100)$ ) [12]. Материал ВFО является перспективным для производства устройств спинтроники, сенсоров и носителей данных. В работе [13] методом резерфордовского обратного рассеяния была изучена диффузия стронция в стекловидном углероде (glassy carbon) при разработке емкости для хранения радиоактивных отходов.

Наряду с этим РОР широко используется в археологии для изучения состава, восстановления внешнего вида и методики производства древних артефактов [1419]. А, например, в работе [20] при помощи данной методики изучался элементный состав метеорита. Так же при помощи РОР решается широкий спектр биомедицинских задач [21]. В работе [22] метод резерфордовской спектрометрии использовался для изучения перспектив использования стронция для применения в регенерации костей.

Анализ создаваемых или модифицируемых ионной имплантацией наноструктур без нарушения условий вакуума позволяет избежать влияния атмосферы на поверхность. Нарушение вакуумных условий стимулирует образование оксидных пленок на поверхности образцов, что может исказить результаты дальнейшего анализа создаваемых ионным облучением структур. Сокращение времени между облучением образца ускоренными ионами и изучением полученных модифицированных структур позволяет минимизировать влияние процессов 


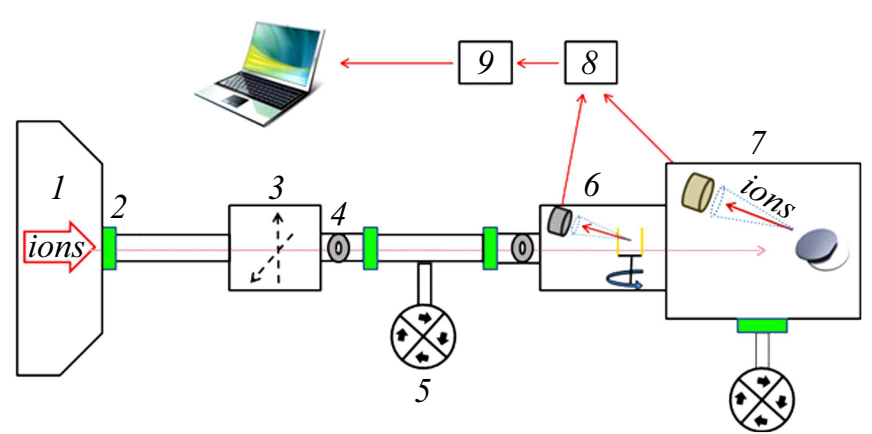

Рис. 1. Схема экспериментального тракта: 1 - направляющий магнит, 2 - вакуумные затворы, 3 - квадрупольная триплетная линза, 4 - прямоугольные диафрагмы, 5 турбомолекулярные насосы, 6 - система мониторирования, 7 - экспериментальная камера, 8 - зарядово-чувствительный предусилитель-формирователь, 9 - усилитель.

диффузии на распределения по глубине внедренной примеси и радиационно-стимулированных дефектов. Преимущества in situ модификации под воздействием ионного облучения мишеней и их анализ ионно-пучковыми методиками реализуются на введенной в эксплуатацию линии, позволяющей проводить облучение мишеней различными типами ионов и изучать создаваемые объекты методом РОР в сочетании с каналированием.

\section{1. Описание экспериментальной установки и методики эксперимента}

Методика ионной имплантации и in situ спектрометрии резерфордовского обратного рассеяния реализована на базе ускорителя ионов на энергии до $500 \mathrm{keV}$. На данной экспериментальной установке можно проводить работы по имплантации однозарядными ионами с массой от 1 до $250 \mathrm{amu}$ с энергией до $500 \mathrm{keV}$, а также исследовать состав, профиль распределения примесей по глубине и совершенство кристаллической структуры мишеней с помощью методики РОР ионами $\mathrm{H}^{+}$или $\mathrm{He}^{+}$ с энергией до $500 \mathrm{keV}$, либо ионами $\mathrm{He}^{++}$с энергией до $1 \mathrm{MeV}$.

Экспериментальная линия состоит из ионопровода, экспериментальной камеры и спектрометрического тракта (рис. 1).

\section{1. Ионопровод}

На рис. 1 представлена схема экспериментального тракта. После прохождения направляющего магнита 1 ускоренный пучок попадает в экспериментальный тракт, где он фокусируется квадрупольной триплетной линзой 3 и при необходимости может быть отклонен по вертикали электродами, на которые подается напряжение до $2.5 \mathrm{kV}$. Далее пучок коллимируется системой из двух прямоугольных диафрагм 4 с регулируемыми размерами (от $0 \times 0$ до $40 \times 40 \mathrm{~mm}^{2}$ ), после чего попадает в экспериментальную камеру 7 через систему мониторирования 6. При размерах двух диафрагм $1 \times 1 \mathrm{~mm}^{2}$, расходимость пучка составляет $0.12^{\circ}$ и растет с увеличением размеров отверстий. Система вакуумной откачки содержит два турбомолекулярных насоса 5 в сочетании с форвакуумными насосами и вакуумные затворы 2. Для быстроты замены образцов предусмотрена возможность предварительной откачки экспериментальной камеры дополнительным форвакуумным насосом. Рабочее давление в камере достигает порядка $10^{-7} \mathrm{mbar}$. Давление остаточных газов измеряется с помощью манометров Пирани (низкий вакуум) и ионизационных вакуумметров (высокий вакуум) с холодным катодом.

\section{2. Экспериментальная камера и измерительный тракт}

Схема экспериментальной камеры и измерительного тракта представлена на рис. 1. Ионы, долетевшие до образца, установленного на гониометрической системе, рассеиваются и регистрируются при помощи кремниевого поверхностно-барьерного детектора.

Заряд, попадающий на исследуемый образец, регистрируется с помощью интегратора тока, однако такой метод не является универсальным. Регистрация заряда, попавшего на образец, усложняется как диэлектрическими свойствами мишеней, так и вторичной электронной эмиссией с поверхности образца, зависящей от параметров пучка и характеристик мишени. Поэтому в случае проведения ионной имплантации регистрация количества падающих на мишень ионов проводится с помощью цилиндра Фарадея, а в случае проведения исследований методом РОР применяется система мониторирования пучка. Спроектированная система мониторирования состоит из вращающейся алюминиевой рамки, на которую методом термического осаждения нанесен тонкий слой золота толщиной $50 \mathrm{~nm}$, и кремниевого поверхностнобарьерного детектора. Рамка приводится в движение гистерезисным двигателем с экранированными полюсами. При вращении рамка периодически перекрывает пучок, при этом детектор регистрирует рассеянные ионы. Из сигналов с детектора пересчитываются те, которые соответствуют ионам, рассеянных от золота. Количество этих импульсов пропорционально количеству ионов падающих на образец. Контролируя количество импульсов системы мониторирования, можно проводить измерения РОР при одинаковом флюенсе облучения анализирующими частицами для различных образцов или участков одного образца.

Импульсы с кремниевого поверхностно-барьерного детектора поступают на зарядово-чувствительный предусилитель-формирователь 8. Зарядово-чувствительный предусилитель-формирователь разработан для того, чтобы обеспечить максимальную разрешающую способность по энергии, при использовании любой выходной цепи. Предусилитель преобразует входной сигнал с детектора в резко возрастающий импульс, спадающий по 


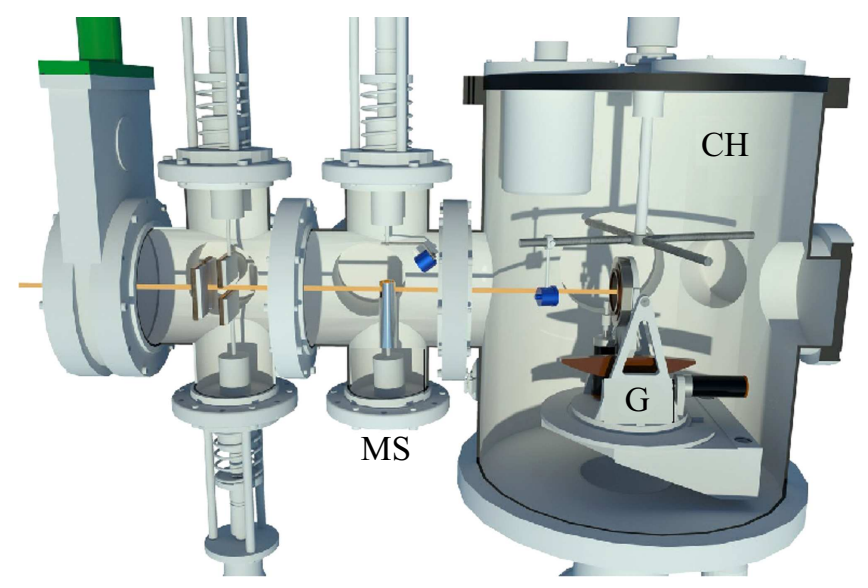

Рис. 2. Гониометр и система мониторирования. $\mathrm{CH}-$ экспериментальная камера, G - гониометр, MS - система мониторирования

экспоненте. Напряжение смещения детектора подается на предусилитель с блока генератора смещения. Также на предусилитель может быть подан сигнал с генератора импульсов для тестирования и калибровки измерительного тракта.

С предусилителя сигнал проходит через усилитель 9, выходные импульсы которого имеют полугауссову форму (semi-Gaussian-shaped), что приводит к уменьшению отношения сигнал/шум и уменьшению времени формирования импульса, что позволяет увеличить скорость счета. После этого сигнал поступает на многоканальный амплитудный анализатор. Используемый анализатор регистрирует импульсы различной амплитуды $\left(5-10^{4} \mathrm{mV}\right)$ и записывает их в соответствующий канал, таким образом, формируя спектр. Далее через последовательный порт спектр передается на компьютер.

Гониометр позволяет осуществлять вращение образца вокруг трех осей и поступательное перемещение в вертикальном направлении, что дает возможность исследовать различные участки поверхности образца без изменения ориентации относительно пучка (рис. 2). Угловые перемещения производятся с помощью шаговых двигателей, причем вращение осуществляется с шагом $0.02^{\circ}$, а вертикальное перемещение с помощью микрометрического винта с точностью $0.1 \mathrm{~mm}$ в диапазоне $20 \mathrm{~mm}$. Точность выполнения поворотов на большие углы не хуже $1 \%$.

Угловое положение детектора относительно мишени можно изменять в плоскости рассеяния по окружности, центр которой совпадает с центром вращения гониометра. Также можно варьировать расстояние детектора от мишени. Управление шаговыми двигателями осуществляется дистанционно с помощью компьютерной программы, разработанной в среде программирования NI LabVIEW.

При работе с монокристаллическими мишенями набор спектра может проводиться в двух режимах: в режиме, при котором ионы движутся в направлении, не содержащем открытых каналов, и в режиме каналирования, когда направление распространения пучка совпадает с направлением одной из кристаллографических осей или плоскостей. Определение направления каналирования происходит следующим образом: образец устанавливается так, чтобы между нормалью к его поверхности и направлением распространения пучка был угол величиной 4-5; образец последовательно отклоняется по углу $\alpha$ (азимутальный угол); каждые 20 шагов шагового двигателя, что соответствует углу $0.4^{\circ}$, образец останавливается и детектор регистрирует спектр обратнорассеянных ионов; из полученного POP спектра выделяется участок, на котором рассчитывается интегральный выход ионов. Из набора спектров, соответствующих серии последовательных отклонений образца на заданное количество шагов, получается кривая выхода обратнорассеянных частиц. Спектры набираются при одинаковом количестве упавших на образец анализирующих частиц. Главный минимум полученной кривой выхода соответствует направлению главной кристаллографической оси. Затем для более точного позиционирования образца относительно пучка производится аналогичное сканирование в области минимума кривой выхода с меньшими интервалами отклонений образца 10 шагов, что соответствует углу $0.2^{\circ}$. Далее гониометр устанавливается в положение, соответствующее наименьшему интегральному выходу по углу $\alpha$. Затем производится такое же сканирование по углу $\theta$ (полярный угол).

\section{2. Определение энергетического разрешения методики}

Для оценки энергетического разрешения методики распространены два метода: использование эталонных

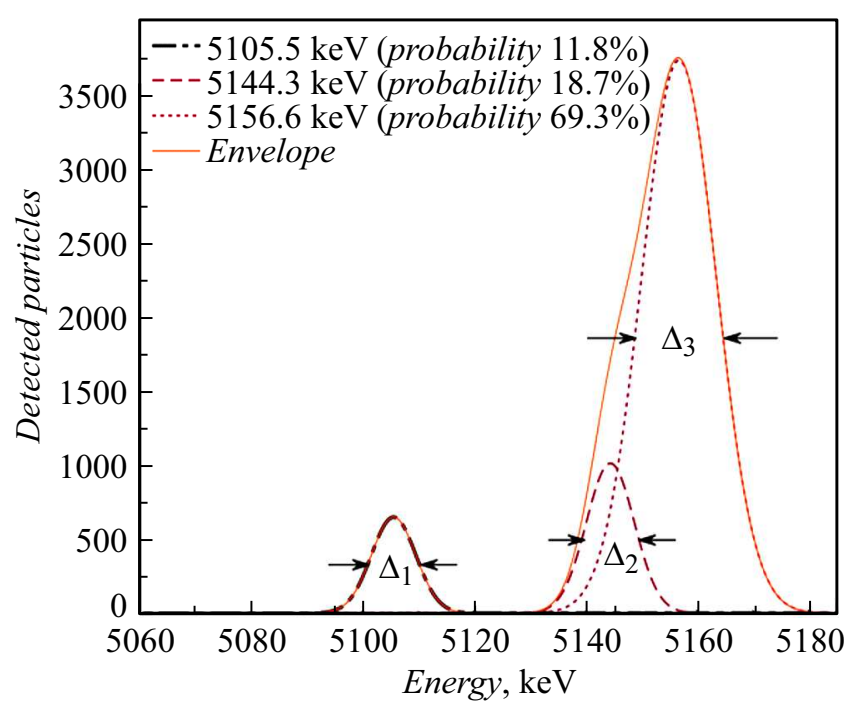

Рис. 3. К определению энергетического разрешения методики: каналы распада ${ }^{239} \mathrm{Pu}$. 
образцов и использование радиоактивных элементов. В качестве эталонных образцов, как правило, используют тонкие пленки, напыляемые на массивные подложки. Этот метод обладает рядом недостатков: начальные этапы процессов напыления и роста пленок являются случайными и поэтому толщина и плотность тонкой пленки могут быть различными на разных участках, так же со временем значительное влияние могут оказать процессы диффузии в образце. Метод, использующий радиоактивные элементы, в этом случае является предпочтительным, поскольку процессы распада хорошо изучены.

Для оценки энергетического разрешения измерительного тракта нами был использован радиоактивный препарат ${ }^{239} \mathrm{Pu}$. Согласно теоретическим данным [23], в спектре ${ }^{239} \mathrm{Pu}$ должны быть различимы три пика: 5156.6 (вероятность 70.77\%), 5144.3 (вероятность 17.11\%), $5105.5 \mathrm{keV}$ (вероятность 11.94\%).

Препарат устанавливался в экспериментальную камеру на место образца. Спектр испускаемых им $\alpha$-частиц записывался при давлении $10^{-5} \mathrm{mbar}$. Энергетическое разрешение измерительного тракта вычислялось по ширине на половине высоты моноэнергетической линии плутония. На рис. 3 представлен полученный спектр.

C помощью стандартных математических процедур [24] было проведено сглаживание экспериментальной кривой и разложение на три пика распада плутония в соответствии с их положением и амплитудой.

Полученные результаты согласуются с теоретическими данными. Однако в данном случае метод позволяет только грубо оценить разрешение методики, так как источник регистрируемого излучения не является точечным, а также в процессе использования источника его поверхность приобрела шероховатость.

Итоговая оценка разрешения методики была сделана при моделировании в программе SIMNRA крутизны спада сигнала, полученного при рассеянии ионов гелия массивным монокристаллом кремния. Разрешение методики по энергии составило $15 \mathrm{keV}$. Для наглядности область спектра, по которой оценивалось разрешение при моделировании, указана на рис. 4. При других значениях разрешения по энергии спад сигнала был либо более вертикальным, либо более пологим.

\section{3. Полученные результаты}

На рис. 4 представлен спектр обратнорассеянных ионов $\mathrm{He}^{++}$с начальной энергией $850 \mathrm{keV}$ от некоторого образца. На спектре в области $500 \mathrm{keV}$ виден сигнал, соответствующий элементу, из которого состоит образец. Определить, что это за элемент, можно, используя законы сохранения энергии и импульса для частиц пучка, рассеивающихся на образце. Пусть пучок сканирующих ионов состоит из частиц массой $M_{1}$ и зарядовым числом $Z_{1}$, частицы обладают энергией $E_{0}$. Частицы пучка сталкиваются с мишенью, которая состоит из атомов

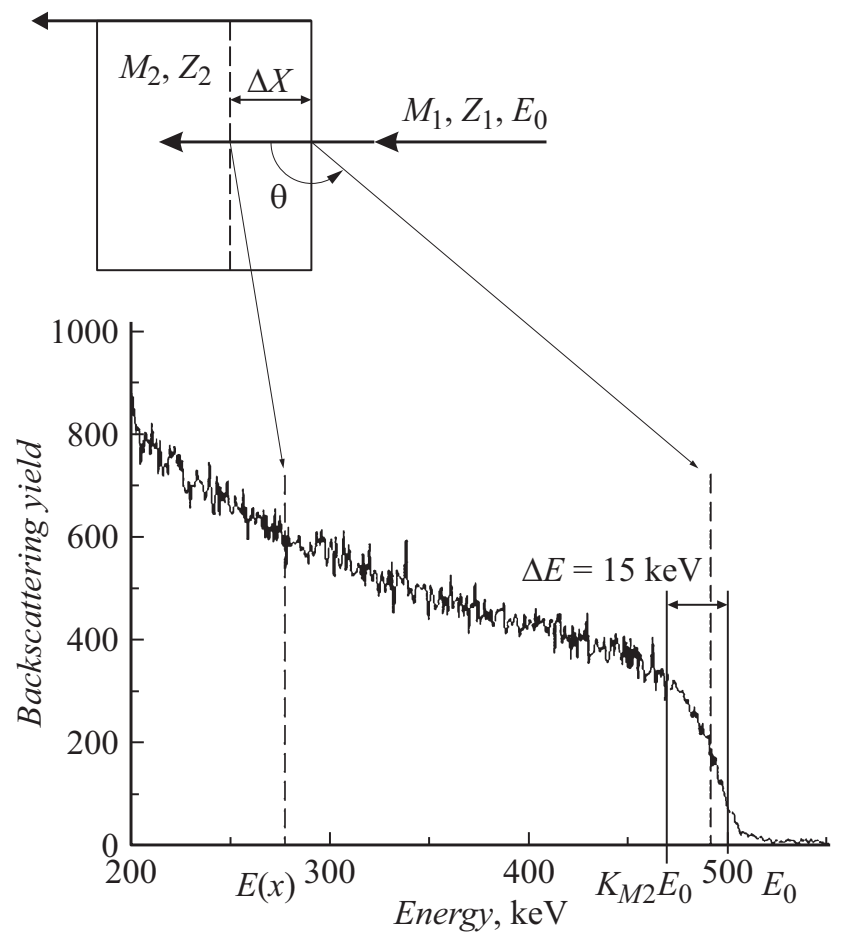

Рис. 4. Энергетический спектр обратно рассеянных ионов $\mathrm{He}^{++}$с энергией $850 \mathrm{keV}$ для угла рассеяния $160^{\circ}$.

массой $M_{2}$. Решая полученную систему уравнений, можно определить отношение энергии $E_{1}$ частицы после упругого рассеяния на угол $\theta$ на атоме мишени $M_{2}$ к ее значению до столкновения $E_{0}$. Это отношение называется кинематическим фактором, обозначается $K_{M_{2}}$ и дается выражением (1) согласно [25]. Таким образом, после рассеяния на атомах, расположенных на поверхности, анализирующие частицы, попадающие в детектор, будут иметь энергию $K_{M_{2}} E_{0}$. А именно зная массу, начальную и конечную энергии частиц анализирующего пучка и угол рассеяния, можно определить элемент, на котором произошло рассеяние, по области спектра, соответствующей началу сигнала от элемента. В данном случае $K_{M_{2}} E_{0} \approx 481 \mathrm{keV}$, что соответствует рассеянию на кремнии.

$$
K_{M_{2}}=\frac{E_{1}}{E_{0}}=\left[\frac{\left(M_{2}^{2}-M_{1}^{2} \sin ^{2} \theta\right)^{\frac{1}{2}}+M_{2} \cos \theta}{M_{1}+M_{2}}\right]^{2} .
$$

Однако на практике чаще всего необходимым является анализ многокомпонентных мишеней. В качестве следующего примера рассмотрим двухкомпонентную мишень, полученную после имплантации ионов $\mathrm{Xe}^{+}$при энергии $100 \mathrm{keV}$ и флюенсе $5 \cdot 10^{15} \mathrm{ion} / \mathrm{cm}^{2}$ в монокристалл кремния (рис. 5).

Исходя из формулы кинематического фактора (1), видно, что, рассеиваясь на атомах поверхности, частицы будут иметь разную энергию, поэтому пики от разных элементов будут располагаться в разных частях спектра. В случае присутствия на поверхности примеси, масса 


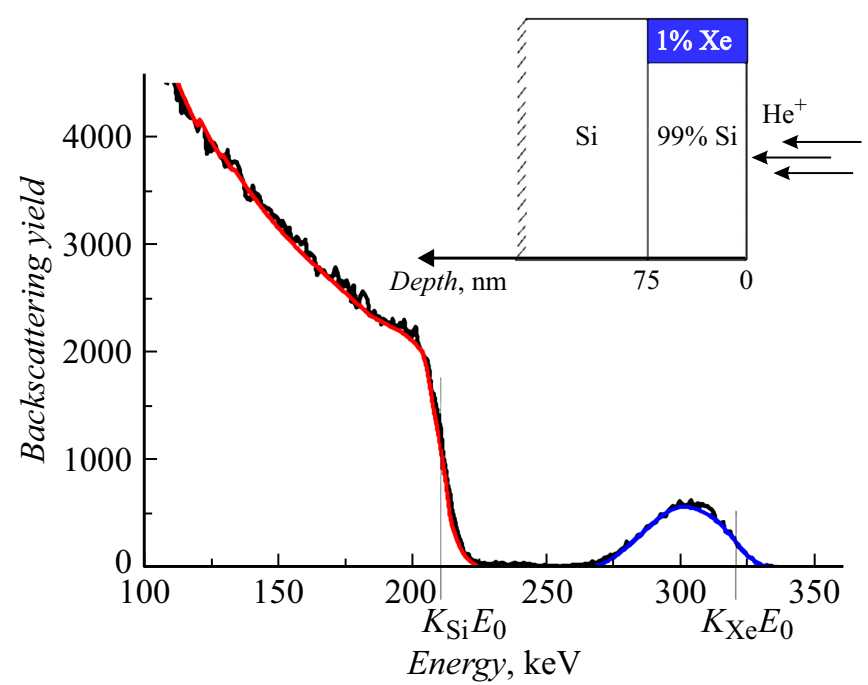

Рис. 5. Энергетический спектр обратно рассеянных ионов $\mathrm{He}^{+}$с энергией $350 \mathrm{keV}$ для угла рассеяния $160^{\circ}$.

атомов которой равна $M_{3}$, на энергетических спектрах обратного рассеяния появится пик в области энергий $K_{M_{3}} E_{0}$. Пик будет расположен в низкоэнергетической области спектра, если $M_{3}<M_{2}$, и в высокоэнергетической, если $M_{3}>M_{2}$. Так, в приведенном примере масса атома ксенона выше массы атома кремния, поэтому $K_{\mathrm{Xe}} E_{0}=311 \mathrm{keV}>K_{\mathrm{Si}} E_{0}=201 \mathrm{keV}$ (приведенные значения энергии соответствуют энергии сканирующих частиц $\left.\mathrm{He}^{+} E_{0}=350 \mathrm{keV}\right)$. Поэтому пик, соответствующий имплантированным ионам ксенона, находится правее части, соответствующей массивному кремнию.

Различие в высотах пиков (что позволяет нам определять относительные концентрации) объясняется разницей в сечении рассеяния на различных элементах. Величина сигнала РОР от химического элемента мишени, или площадь под пиком определяется выражением (2) согласно [25]:

$$
A=q \Omega \sigma(\theta) N_{x},
$$

где $N_{x}-$ поверхностная концентрация элемента $\left(1 / \mathrm{cm}^{2}\right), \sigma$ - среднее дифференциальное сечение рассеяния анализирующих частиц на атомах мишени в детектор с телесным углом $\Omega\left(\mathrm{cm}^{2} / \mathrm{sr}\right), q-$ полное число анализирующих частиц, попавших в мишень за время измерения спектра. Из соотношения (2) следует, что при стандартных условиях эксперимента (т.е. при постоянных $\Omega$ и $q$ ) величина сигнала пропорциональна $\sigma$. Для вычисления среднего дифференциального сечения можно воспользоваться формулой (3) согласно [25]:

$$
\sigma \propto\left(\frac{Z_{1} Z_{2} e^{2}}{4 E \sin ^{2} \theta / 2}\right)^{2} .
$$

Из последней формулы следует, что величина сигнала в спектрах обратного рассеяния зависит от порядкового номера элемента как $Z^{2}$.
Согласно спектру РОР (рис. 5), максимум сигнала от ксенона и от кремния относятся как $A_{\mathrm{Xe}} / A_{\mathrm{Si}}=0.28$. Поскольку энергии анализирующих частиц и угол расположения детектора для обоих веществ совпадают, то отношение сечений рассеяния можно рассматривать как отношение квадратов зарядовых чисел: $\sigma_{\mathrm{Xe}} / \sigma_{\mathrm{Si}}=Z_{\mathrm{Xe}}^{2} / Z_{\mathrm{Si}}^{2}=14.9$. Далее, используя формулу (2) и приведенные числовые отношения, можно определить относительные атомные концентрации ксенона и кремния, соответствующие максимуму распределения примеси: $N_{\mathrm{Xe}} / N_{\mathrm{Si}}=\left(Z_{\mathrm{Si}}^{2} / Z_{\mathrm{Xe}}^{2}\right) \cdot\left(A_{\mathrm{Xe}} / A_{\mathrm{Si}}\right)=0.02$. А именно, согласно вычислениям, максимальная концентрация примеси составляет $2 \%$, средняя атомная концентрация ксенона в модифицированном слое составляет 1\%.

По ширине сигнала РОР определяется толщина слоя, в котором распределена внедренная примесь. Для этого регистрируется разница в энергии $\Delta E$ анализирующих частиц, рассеянных атомами мишени с разной глубины. Шкала глубины определяется энергетическими потерями $d E / d x$ анализирующих ионов при их прохождении через образец. Эти потери пропорциональны пройденной ионами толщине вещества, данные о потерях можно найти в [26]. Таким образом, из спектра РОР приведенного на рис. 5, было определено, что после имплантации ионы ксенона проникли на глубину 75 nm, а их средняя концентрация в слое составила $1 \%$. Для аналогичных расчетов используются программы SIMNRA, Rump.

Согласно формуле для сечения рассеяния (3), выход обратнорассеянных ионов $\mathrm{He}^{+}$зависит от зарядового числа атома мишени и энергии анализирующих частиц. Соответственно чем меньше концентрация примеси, тем больше флюенс облучения ионами $\mathrm{He}^{+}$в процессе РОР анализа необходим для достижения приемлемой точности. На рис. 6 представлены профили распределения ионов ксенона, имплантированных в кремний с флюенсом $5 \cdot 10^{14} \mathrm{ion} / \mathrm{cm}^{2}$, при различных флюенсах $\mathrm{He}^{+}$в процессе проведения анализа методом РОР. При аппроксимации распределением Гаусса статистическая ошибка составила $28,13,3 \%$ соответственно для флюенсов анализирующих частиц $\mathrm{He}^{+} 3 \cdot 10^{11} \mathrm{ion} / \mathrm{cm}^{2}$, $1.25 \cdot 10^{12} \mathrm{ion} / \mathrm{cm}^{2}, 10^{13} \mathrm{ion} / \mathrm{cm}^{2}$.

К возможностям данного метода так же относится снятие спектра в режиме каналирования, что позволяет исследовать качество кристаллической структуры образца.

Атомы кристаллической решетки вдоль основных кристаллографических направлений формируют каналы. Каждый из них ограничивается несколькими плотноупакованными атомными рядами. Частица, которая попала в такой канал, может проникать значительно глубже, чем при движении в направлении, не содержащем открытых каналов. Это связано с тем, что такая частица при движении в канале испытывает только скользящие соударения с его стенками [27] (рис. 7). Критический угол каналирования, т.е. максимальный угол отклонения траектории частицы от кристаллографического направления в кристалле, при котором еще возможно каналирование, 


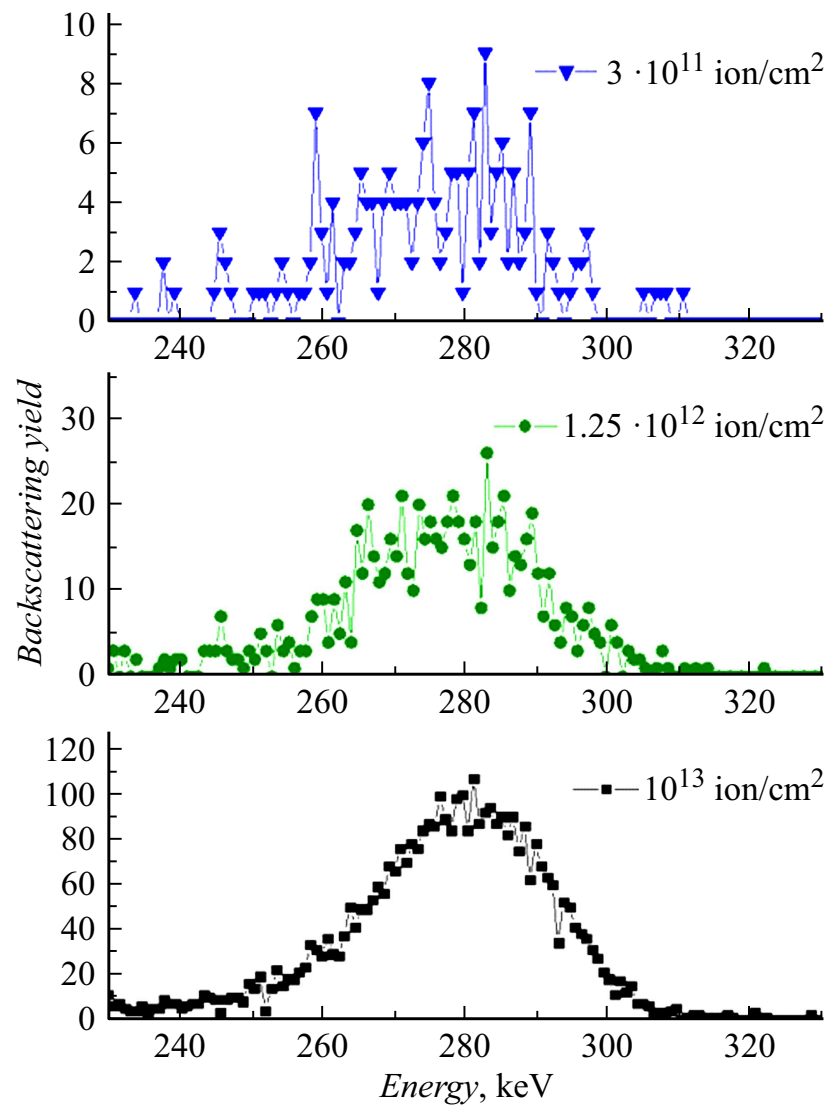

Рис. 6. Профили распределения внедренных в кремний ионов ксенона с энергией $100 \mathrm{keV}$ и флюенсе $5 \cdot 10^{14} \mathrm{ion} / \mathrm{cm}^{2}$, полученные при различных флюенсах анализирующих частиц.

определяется следующим образом согласно $[25,27]$ :

$$
\psi_{c}=\left(\frac{U\left(r_{\min }\right)}{E}\right)^{1 / 2},
$$

где $U(r)$ - атомный потенциал, $r_{\min }-$ максимальное расстояние сближения налетающей частицы с атомом мишени, при котором происходит упругое рассеяние, $E-$ энергия пучка. Тогда характеристический угол каналирования можно рассчитать по формуле (5) согласно [28]:

$$
\psi_{c}=\left(\frac{2 Z_{1} Z_{2} e^{2}}{d E}\right)^{1 / 2},
$$

где $d-$ расстояние между соседними атомами в цепочке.

Используя приведенные формулы, получаем, что при облучении монокристалла кремния ионами гелия с энергией $350 \mathrm{keV}$ критический угол каналирования $\psi_{c}$ для грани (110) с $d=3.84 \AA$ составил $1.4^{\circ}$.

При исследовании образцов методом РОР в направлении каналирования выход обратнорассеянных ионов резко уменьшается примерно на два порядка в результате того, что каналированные частицы не могут подойти достаточно близко к атомным ядрам и подвергнуться рассеянию на большой угол [25]. Отношение величины этого выхода $Y_{1}$ к уровню сигнала, измеренному для углов больше критического $Y_{2}$, т.е. в отсутствие каналирования, называют минимальным выходом $\chi_{\text {min }}$ (рис. 7). Чем ниже значение $\chi_{\min }$, тем выше качество кристаллической структуры образца. Зависимость выхода рассеянных частиц от угла является симметричной функцией с минимумом. И ширина на половине высоты этого распределения соответствует величине $2 \psi_{c}$. Для распределения на рис. 7 эта величина составляет $2.7^{\circ}$, что находится в хорошем согласии со значением, полученным по формуле (5).

Для оценки влияния облучения анализирующими частицами $\mathrm{He}^{+}$на кристаллическую структуру образца

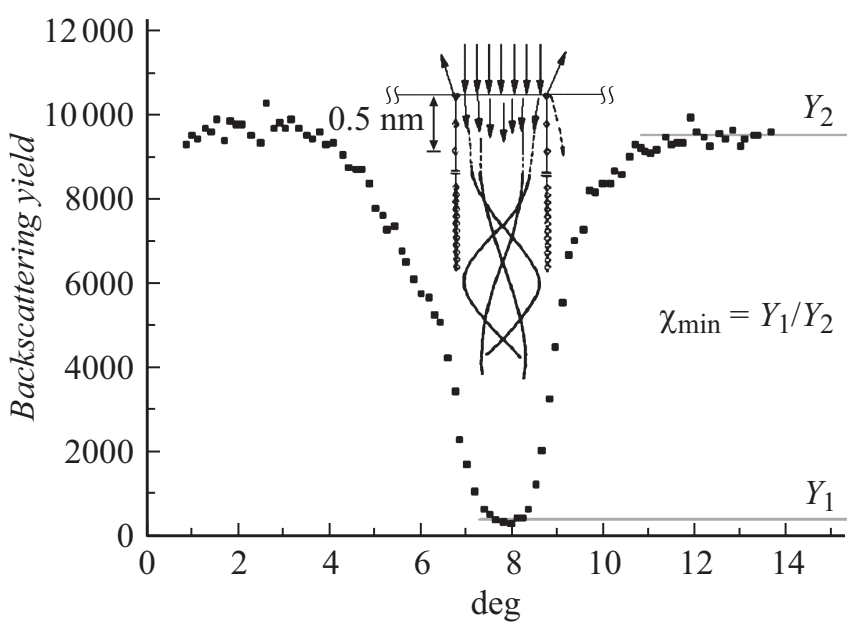

Рис. 7. Зависимость интегрального выхода рассеянных частиц от положения гониометра при вращении по углу $\alpha$.

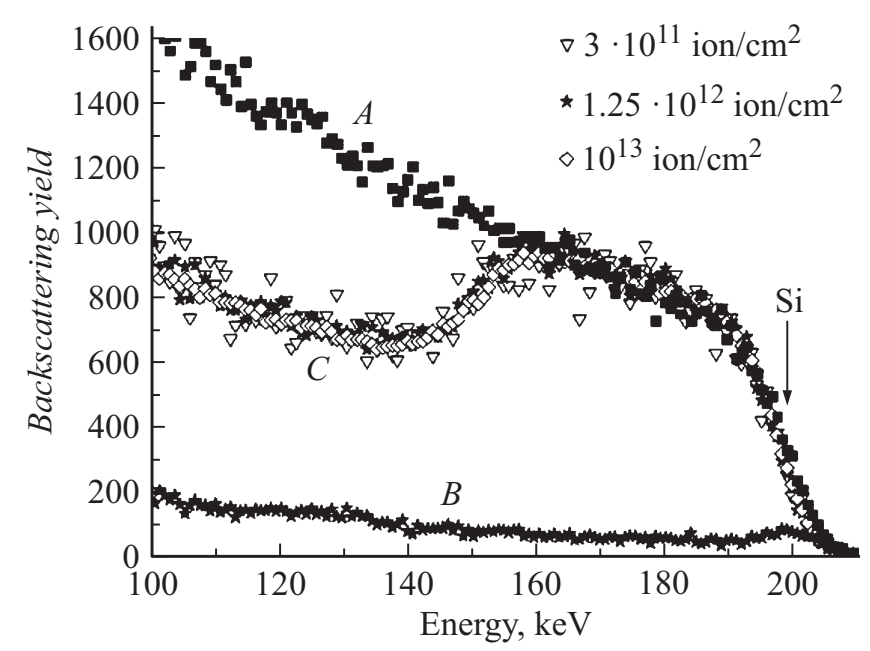

Рис. 8. Энергетический спектр обратно рассеянных ионов $\mathrm{He}^{+}$с энергией $350 \mathrm{keV}$ для угла рассеяния $160^{\circ}$ после имплантации ионов $\mathrm{Xe}^{+}$с энергией $100 \mathrm{keV}$. Спектр исходной структуры: $A-$ в направлении, не содержащем открытых каналов; $B-$ в направлении каналирования. Спектр после имплантации: $C-$ в направлении каналирования для различных доз анализирующих частиц. 
Si сняты спектры POP в режиме каналирования при различных флюенсах. На рис. 8 представлены спектры РОР, полученные от чистого монокристалла кремния (кривая $B$ ), и от монокристалла кремния после облучения ионами ксенона с энергией $100 \mathrm{keV}(A$ и $C)$. Кривая $A$ была снята в режиме, когда направление пучка сканирующих частиц не совпадает с направлением каналов в монокристалле, сигнал от кремния на этом спектре одинаков для исходного и облученного образца. Кривые $B$ и $C$ сняты в режиме каналирования. Как говорилось выше, при попадании пучка частиц в канал их выход рассеяния резко падает, что мы и видим при сравнении кривых $A$ и $B$, соответствующих исходной структуре. Небольшой пик на кривой $B$ в области $190-210 \mathrm{keV}$ соответствует рассеянию на атомах поверхности. Если сравнить кривые $A$ и $C$, соответствующие структуре после облучения, то мы видим полное совпадение кривых в районе $170-210 \mathrm{keV}$, что говорит о полном разрушении кристаллической решетки на всей глубине проникновения ионов ксенона.

Если подробно рассмотреть спектры типа $C$, полученные при разных флюенсах анализирующих частиц, видно, что в процессе РОР анализа при указанных условиях увеличение флюенса $\mathrm{He}^{+}$на два порядка не приводит к изменению профиля дефектов.

\section{Заключение}

Метод РОР в сочетании с каналированием позволяет изучать состав и структуру мишени. Спектрометрия РОР достаточно хорошо отработана и широко применяется в исследованиях различных направлений и дисциплин. В настоящей работе продемонстрирована возможность применения методики для изучения состава и структуры монокристаллических мишеней, подвергнутых ионному облучению при различных параметрах пучка. Реализованная методика позволяет с высоким разрешением по глубине получать профили внедренной примеси и образовавшихся дефектов кристаллической структуры, а также с высокой чувствительностью по концентрации определять элементный состав образца. Замещение атомов в узлах решетки, наличие и качество кристаллического слоя, формирование аморфного слоя также определяются как функция глубины.

В настоящей работе было показано, что анализ концентрации примеси и дефектов кристаллической структуры с погрешностью на уровне нескольких процентов достигается при флюенсе облучения анализирующими частицами порядка $10^{13} \mathrm{ion} / \mathrm{cm}^{2}$. Было показано, что в процессе анализа при указанных флюенсах не происходит разрушение структуры мишени под действием потока анализирующих частиц.

Работа выполнена при поддержке Министерства образования и науки РФ (соглашение № 14.581.21.0021, уникальный идентификатор соглашения

\section{Список литературы}

[1] Horodek P., Siemek K., Kobets A.G., Kulik M., Meshkov I.N. // Appl. Surf. Sci. 2015. Vol. 333. P. 96-103

[2] Lenka H., Meersschaut J., Kandaswamy P.K., Modarresi H., Bender H., Vantomme A., Vandervorst $W$. // Nucl. Instrument. Method. Phys. Research, Section B: Beam Interactions with Materials and Atoms. 2014. Vol. 331. P. 69-73.

[3] Kummari V.C., Reiner T., Jiang W., McDaniel F.D., Rout F.D. // Nucl. Instrument. Method. Phys. Research, Section B: Beam Interactions with Materials and Atoms. 2014. Vol. 332. P. $28-32$

[4] Hallén A., Moschetti G. // Nucl. Instrument. Method. Phys. Research, Section B: Beam Interactions with Materials and Atoms. 2014. Vol. 332. P. 172-175.

[5] Chuan-Lei Jia, Zhi-Ning Wei // Physica B. 2014. Vol. 37. P. 1-3.

[6] Wendler E., Becker G., Rensberg J., Schmidt E., Wolf S., Wesch W. // Nucl. Instrument. Method. Phys. Research, Section B: Beam Interactions with Materials and Atoms. 2016. Vol. 379. P. 195-199.

[7] Shemukhin A.A., Nazarov A.V., Balakshin Yu.V., Chernysh V.S. // Nucl. Instrument. Method. Phys. Research, Section B: Beam Interactions with Materials and Atoms. 2015. Vol. 354. P. 274-276.

[8] Шемухин А.А., Балакшин Ю.В., Черныш В.С., Голубков С.А., Егоров Н.Н., Сидоров А.И. // ФТП. 2014. Т. 48. Вып. 4. С. 535-538. [Shemukhin A.A., Balakshin Y.V., Chernysh V.S., Golubkov S.A., Egorov N.N., Sidorov A.I. // Semiconductors. 2014. Vol. 48. P. 517-520.]

[9] Shemukhin A.A., Balakshin Yu.V., Evseev A.P., Chernysh V.S. // Nucl. Instrument. Method. Phys. Research, Section B: Beam Interactions with Materials and Atoms. 2017. Vol. 406. P. 507-510.

[10] Moloi S.J., McPherson M. // Vacuum. 2014. Vol. 104. P. 51-56.

[11] Chiang H.-Y., Park S.-H., Mayer M., Schmid K., Balden M., Boesenberg U., Jungwirth R., Falkenberg G., Zweifel T., Petry W. // J. Alloys Compd. 2015. Vol. 626. P. 381-390.

[12] Xiao R.-Z., Wang Z.-S., Yuan X.-B., Zhou J.-J., Mao Z.-L., Su H.-S., Li B., Fu D.-J. // Nucl. Instrument. Method. Phys. Research, Section B: Beam Interactions with Materials and Atoms. 2016. Vol. 384. P. 106-112.

[13] Odutemowo O.S., Malherbe J.B., Theron C.C., Njoroge E.G., Wendler E. // Vacuum. 2016. Vol. 126. P. 101-105.

[14] Albéric M., Müller K., Pichon L., Lemasson Q., Moignard B., Pacheco C., Fontan E., Reiche I. // Talanta. 2015. Vol. 137. P. $100-108$.

[15] Santos H.C., Added N., Silva T.F., Rodrigues C.L. // Nucl. Instrument. Method. Phys. Research, Section B: Beam Interactions with Materials and Atoms. 2015. Vol. 345. P. 42-47.

[16] Fourdrin C., Camagna S.P., Pacheco C., Radepont M., Lemasson Q., Moignard B., Pichon L., Bourgeois B., Jeammet V. // Microchem. Journ. 2016. Vol. 126. P. 446-453.

[17] Ortega-Feliu I., Ager F.J., Roldán C., Ferretti M., Juanes D., Scrivano S., Respaldiza M.A., Ferrazza L., Traver I., Grilli M.L. // Nucl. Instrument. Method. Phys. Research, Section B: Beam Interactions with Materials and Atoms. 2017. Vol. 406. P. 318-323. 
[18] Beck L., Alloin E., Vigneron A., Caffy I., Klein I. // Nucl. Instrument. Method. Phys. Research, Section B: Beam Interactions with Materials and Atoms. 2017. Vol. 406. Pt. A. P. 93-98.

[19] Wu Q.Q., Zhu J.J., Liu M.T., Zhou Z., An Z., Huang W., He Y.H., Zhao D.Y. // Nucl. Instrument. Method. Phys. Research, Section B: Beam Interactions with Materials and Atoms. 2013. Vol. 296. P. 1-6.

[20] Noun M., Roumie M., Calligaro T., Nsouli B., Brunetto R., Baklouti D., d'Hendecourt L., Della-Negra S. // Nucl. Instrument. Method. Phys. Research, Section B: Beam Interactions with Materials and Atoms. 2013. Vol. 306. P. $261-264$.

[21] Ren M.Q., Ji X., Vajandar S.K., Mi Z.H., Hoi A., Walczyk T., van Kan J.A., Bettio A.A., Watt F., Osipowicz T. // Nucl. Instrument. Method. Phys. Research, Section B: Beam Interactions with Materials and Atoms. 2017. Vol. 406 Pt. A. P. $15-24$.

[22] Lacroix J., Lao J., Nedelec J.-M., Jallot E. // Nucl. Instrument. Method. Phys. Research, Section B: Beam Interactions with Materials and Atoms. 2013. Vol. 306. P. 153-157.

[23] National Nuclear Data Center, Brookhaven, USA http://www.nndc.bnl.gov/chart/decaysearchdirect.jsp?nuc $=239$ PU\&unc $=$ nds

[24] Электронный ресурс. Режим доступа: https://www.originlab.com/

[25] Nastasi M., Mayer J.W., Wang Y. Ion Beam Analysis: Fundamentals and Applications. London: CRC Press, 2015. $460 \mathrm{p}$.

[26] Электронный ресурс. Режим доступа: http://www.exphys.uni-linz.ac.at/Stopping/

[27] Титов В.В. Внедрение быстрых ионов в монокристаллы. М.: ИЭА, 1978. $35 \mathrm{c.}$

[28] Elich J.J.Ph., Roosendaal H.E., Onderdelinden D. // Radiat eff Defects Solids. 1971. Vol. 10. P. 175-184. 